UNIVERSIDADE FEDERAL DE SÃO PAULO

CAMPUS BAIXADA SANTISTA

DEPARTAMENTO DE CIÊNCIAS DO MAR

ENGENHARIA DE PETRÓLEO

MODELAGEM NUMÉRICA PARA GERAÇÃO DE SÉRIES SINTÉTICAS DE IRRADIÂNCIA SOLAR MINUTO A MINUTO

Thaiane Gambarra Soares

Santos, 2018 


\section{MODELAGEM NUMÉRICA PARA GERAÇÃO DE SÉRIES SINTÉTICAS DE IRRADIÂNCIA SOLAR MINUTO A MINUTO}

\section{THAIANE GAMBARRA SOARES}

Trabalho de conclusão de curso submetido ao curso de Engenharia de Petróleo, como requisito parcial para a obtenção do título de Engenheira de Petróleo. Orientador: Prof. Dr. Fernando Ramos Martins. 
Dedico este trabalho ao meu marido Igor, que com seu amor e exemplo sempre me incentivaram a buscar meus sonhos. Amo você. 


\section{AGRADECIMENTOS}

Ao meu marido Igor que sempre me apoia e me ajuda de maneiras até mesmo impossíveis em todos os momentos da minha vida por todos esses anos de amor e companheirismo.

Ao meus pais, Teresa e Amílcar e aos meus irmãos Amílcar Júnior e Thaise pois sem seu apoio e exemplo eu nunca teria chegado onde estou.

Ao meu orientador Prof. Dr. Fernando Ramos Martins pela paciência, ajuda e ensinamentos durante a elaboração desta monografia. Obrigada por sempre ter se mostrado muito solícito e acolhedor.

Ao Dr. Francisco Lima que contribuiu para o trabalho durante o desenvolvimento do algoritmo e no cálculo do modelo de céu claro.

Às minhas amigas Àvylla, Paula Flávia, Ana Cláudia e Juliana por deixarem esta etapa da minha vida mais feliz com tantas risadas. Obrigada pelo companheirismo e pela amizade. 


\section{RESUMO}

Um dos maiores desafios para o atendimento da demanda de energia será a integração em larga escala de fontes de energias renováveis intermitentes às redes de distribuição de eletricidade. As fontes de energias renováveis, como o Sol e os ventos possuem um caráter flutuante espacial e temporal intrínsecos aos processos físicos da natureza. Sua utilização de forma eficiente na geração de eletricidade deve conter sistemas operacionais para distribuição que considerem essa variabilidade. As companhias de distribuição devem lidar com dificuldades associadas às variações de entrada e com sobrecargas ou paradas no sistema de distribuição, para tanto um modelo confiável de geração de uma série temporal de radiação solar é bastante útil. Este trabalho teve como objetivo adaptar um modelo de previsão de irradiância global de minuto a minuto a partir de uma base de dados horários com finalidade de possibilitar melhor simulação do aproveitamento da energia solar em projetos de exploração e comercialização desta fonte. $\mathrm{O}$ estudo tomou como base o modelo estatístico desenvolvido por Hofmann et al. (2014) fundamentado em cadeias de Markov. Matrizes de probabilidade de transição (MPT) foram geradas para as seguintes condições de nebulosidade: nublado, parcialmente nublado ou céu claro. As matrizes foram determinadas utilizando uma série temporal de dados horários de irradiância solar com dois anos de dados horários observados na estação de superfície (INPE/SONDA) localizada em São Martinho da Serra - RS. A partir das matrizes MPT, utilizou-se o método da transformada inversa para a geração de uma série de dados com resolução temporal de um minuto levando em consideração a classificação da condição de nebulosidade dos dados horários de entrada. O modelo produzido no estudo tem aplicação local, mas a metodologia empregada pode ser replicada para qualquer localidade no Brasil. A validação do modelo foi realizada utilizando dados de irradiância solar medidos na estação meteorológica não utilizados na elaboração das MPTs. O erro quadrático médio das frequências de distribuição relativa indicou um desvio de apenas 0,015 e o teste de Kolmogorov-Smirnov não rejeitou a hipótese de que as séries temporais observada e gerada pelo modelo apresentam a mesma distribuição de frequência cumulativa. Desta forma, o modelo estatístico desenvolvido apresentou uma alta confiabilidade em reproduzir a variabilidade temporal decorrente da natureza da energia solar no local de estudo. 


\section{LISTA DE ILUSTRAÇÕES}

Figura 1. Movimento da Terra em relação ao Sol. (Fonte: IQBAL, 1983). 10

Figura 2. Espectro de radiação solar incidente no topo da atmosfera e na superfície da Terra e da emissão de um corpo negro a 5900 K. Fonte: MARTINS et al., 2004. 12

Figura 3. Curso temporal da irradiância global para três condições de tempo diferentes. (a) Condição de céu nublado. (b) Condição de céu parcialmente nublado. (c) Condição de céu claro. A linha vermelha corresponde a valores de irradiância global medidos (dado de validação) e a linha azul corresponde a valores de irradiância global gerados a partir do modelo estatístico desenvolvido neste trabalho. 26

Figura 4. Frequência de gradientes da irradiância global em São Martinho da Serra RS. A linha azul corresponde a frequência de gradientes gerados pelo modelo estatístico e a linha vermelho corresponde a frequência dos dados medidos na estação. 27

Figura 5. Frequência anual de distribuição relativa da irradiância global. Linha em azul representa os dados modelados e a linha em vermelho representa o dado medido 28

Figura 6. Frequência de distribuição acumulada anual. Em azul encontram-se os dados do modelo estatístico desenvolvido e em vermelho encontram-se os dados medidos na estação meteorológica 


\section{LISTA DE TABELAS}

Tabela 1. Visão geral das três classes de tempo e suas condições de detecção 20

Tabela 2. Fragmento da MPT para a condição de céu nublado. Para cada valor de Kt no instante de tempo i (linhas), a probabilidade de mudança para outro valor de Kt no instante de tempo i+1 (colunas) é dado. Os valores de Kt variam de 0 a 1,5............... 25 Tabela 3. Desvios médios quadráticos (RMSE) das frequências de distribuição de gradientes de irradiância e das frequências relativas de distribuição em porcentagem dos dados modelados quando comparados aos dados medidos. 28 


\section{SUMÁRIO}

1. INTRODUÇÃ

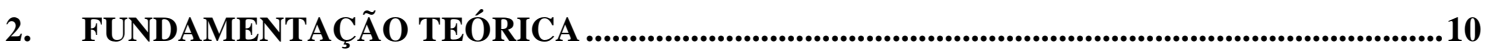

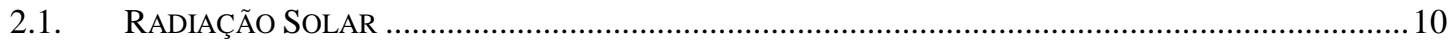

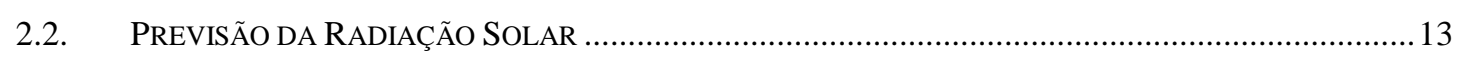

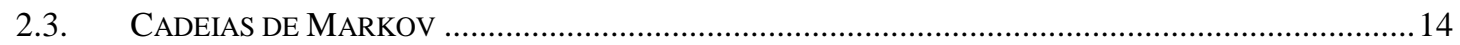

2.3.1. Definição da Cadeia de Markov …………….......................................................... 15

2.3.2. Método da Transformada Inversa ................................................................................ 15

3. OBJETIVOS ….............................................................................................................................

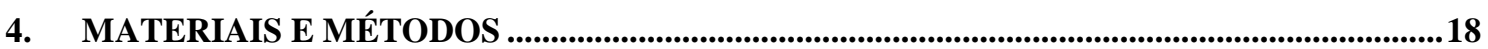

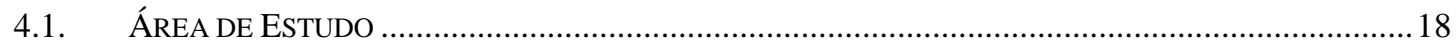

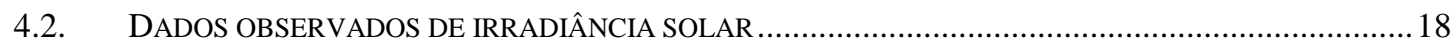

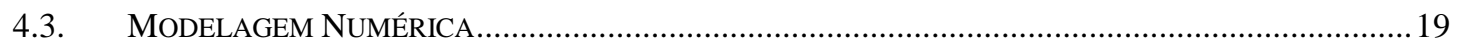

4.3.1. Matriz de Transição de Probabilidade (MPT) ............................................................... 19

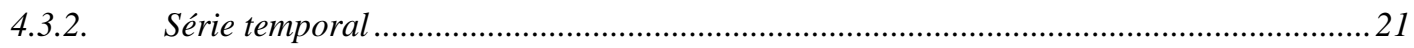

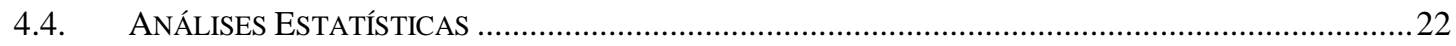

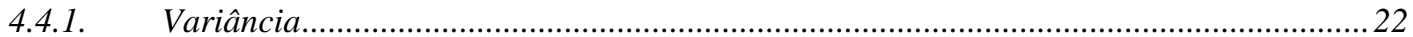

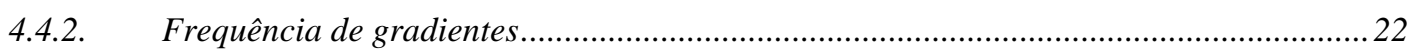

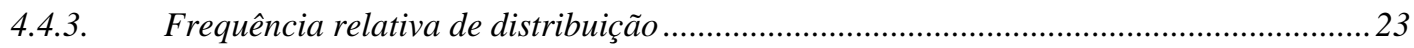

4.4.4. Teste de Kolmogorov-Smirnov ................................................................................2

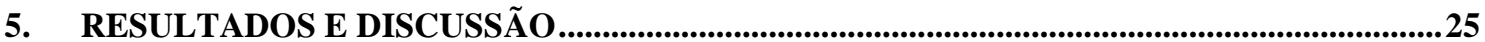

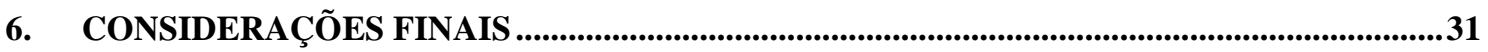

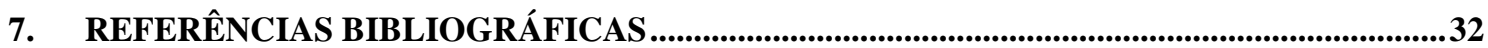




\section{INTRODUÇÃO}

O Sol é fonte de energia renovável e inesgotável; e o aproveitamento dessa energia é uma das alternativas energéticas mais promissoras no Brasil. Nosso país possui altos níveis de insolação e grandes reservas de quartzo de qualidade, matéria-prima utilizada na fabricação de painéis fotovoltaicos para conversão de energia solar em eletricidade (EPE, 2012).

Um dos maiores desafios para o atendimento da demanda de energia será a integração em larga escala de fontes de energias renováveis intermitentes às redes de distribuição de eletricidade. As fontes de energias renováveis, como o Sol e os ventos possuem um caráter flutuante espacial e temporal intrínsecos aos processos físicos da natureza e a sua utilização de forma eficiente para converter energia em eletricidade deve conter sistemas operacionais para distribuição de eletricidade que considerem essa variabilidade (HEINEMANN et al., 2006).

A problemática da variabilidade da incidência de radiação solar é um fator que deve ser analisado com bastante cuidado tanto do ponto de vista econômico quanto do ponto de vista de segurança do sistema elétrico. As companhias de distribuição devem lidar com dificuldades associadas às variações de entrada e com sobrecargas ou paradas no sistema de distribuição, para tanto um modelo confiável de previsão de radiação solar é bastante útil (DIAGNE et al., 2013).

A maioria dos dados de irradiância solar comerciais ou mesmo aqueles disponíveis gratuitamente (Meteotest, SolarGIS, TMY e Atlas Brasileiro de Energia Solar) encontram-se com valores instantâneos de hora em hora. Todavia para estimar a quantidade de irradiação de uma determinada área e posteriormente realizar o dimensionamento de uma planta fotovoltaica de uma forma mais precisa seriam necessários dados de minuto a minuto de irradiância solar. O objetivo deste trabalho foi criar um modelo estatístico que utiliza como dados de entrada valores horários de irradiância solar e gera dados de minuto a minuto. 


\section{FUNDAMENTAÇÃO TEÓRICA}

\subsection{Radiação Solar}

A energia proveniente do Sol, denominada de Radiação Solar é sujeita à várias interações na atmosfera até chegar à superfície terrestre. As interações são provenientes das características geométricas da relação Sol-Terra, da distribuição espectral do fluxo solar extraterrestre e dos fenômenos provocados por processos radiativos com a atmosfera.

A Terra se move em um plano fixo ao redor do Sol e a órbita da Terra é uma elipse onde o Sol está situado em um de seus focos. A quantidade de radiação solar que incide sobre a Terra é inversamente proporcional ao quadrado da distância Sol-Terra. A distância média é de $1 \mathrm{AU}\left(1,496 \times 10^{8} \mathrm{~km}\right)$, entretanto com o movimento ao longo do ano, essa distância varia, sendo seu valor mínimo denominado periélio (0,983 AU) e seu valor máximo afélio (1,017 AU) (IQBAL, 1983) (Figura 1).

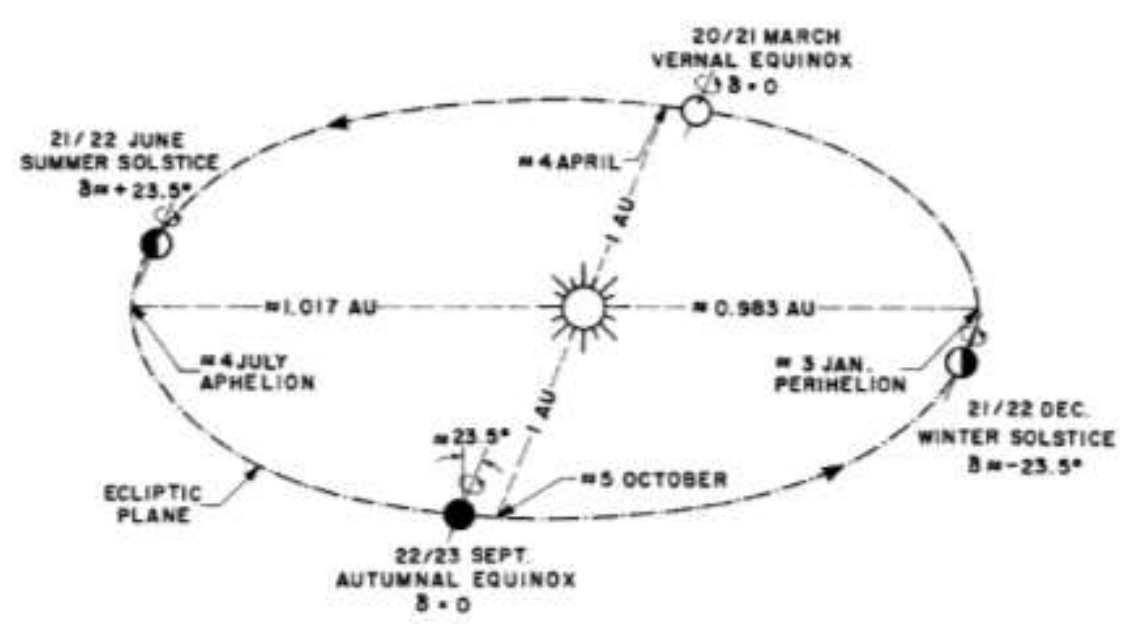

Figura 1. Movimento da Terra em relação ao Sol. (Fonte: IQBAL, 1983).

As estações do ano existem devido não só ao movimento de translação da Terra em relação ao Sol, como também devido à inclinação do eixo de rotação da Terra em relação à linha perpendicular do plano elíptico. O valor do ângulo de inclinação é de $23,45^{\circ}$ e permanece constante durante todo o percurso de translação da Terra.

Os equinócios ocorrem quando a duração do dia e da noite são iguais, em qualquer ponto da Terra. A radiação solar incidente com a mesma intensidade tanto no hemisfério Norte como Sul. Nos solstícios, há uma diferença máxima entre a duração do dia e da 
noite, sendo que, a noite será a maior do ano em um hemisfério enquanto que, no outro hemisfério, a noite terá a menor duração. Os solstícios correspondem à passagem da Terra pelo seus pontos em que a radiação solar incide perpendicularmente sobre um dos trópicos de Capricórnio ou Câncer.

Além do movimento de translação, a Terra também realiza o movimento de rotação em torno de seu próprio eixo, determinando o início e o final de um dia. Devido às características de translação, rotação e inclinação, regiões do globo terrestre recebem os raios do Sol com ângulos de incidência diferentes, segundo a hora do dia e a época do ano, e que eles se modificam com a orientação e a inclinação em relação ao plano da órbita terrestre.

O Sol emite energia na forma de radiação eletromagnética, este tipo de radiação não necessita de um meio material para se propagar. A energia proveniente do Sol pode ser relacionada pela Lei de Planck:

$$
E=\frac{h c}{\lambda}
$$

onde $\mathrm{h}$ é a constante de Planck $\left(6,6 \times 10^{-34} \mathrm{~J} . \mathrm{s}\right)$, c é a velocidade da luz no vácuo $(3 \mathrm{x}$ $10^{8} \mathrm{~m} / \mathrm{s}$ ) e $\lambda$ é o comprimento da onda eletromagnética.

A radiação eletromagnética existe para um grande intervalo de comprimentos de onda e está dividida em bandas ou faixas espectrais. $\mathrm{O}$ espectro da radiação solar tem a maior parte de sua energia contida em comprimentos de onda entre 0,1 e $4 \mu \mathrm{m}$, e é comumente dividido em três faixas principais: ultravioleta $(0,1 \leq \lambda \leq 0,4 \mu \mathrm{m})$, visível $(0,4<\lambda \leq 0,7 \mu \mathrm{m})$ e infravermelho $(>0,7 \mu \mathrm{m})(\mathrm{IQBAL}, 1983)$.

O fluxo total da irradiação solar extraterrestre medido fora da atmosfera terrestre e integrado sob todo o espectro solar é denominado Constante Solar $\left(\mathrm{S}_{\mathrm{o}}\right)-$ fluxo de energia solar por unidade de tempo e de área normal à irradiação, para uma distância média Terra/Sol. Seu valor recomendado pelo WRC (World Radiation Center) é de $1367 \mathrm{~W} / \mathrm{m}^{2}$. Devido às ligeiras variações da distância Terra-Sol ao longo do ano, decorrentes da excentricidade da órbita terrestre, a irradiância solar que atinge o topo da atmosfera (TOA) sofre alterações (DUFFIE e BECKMAN, 1991) que podem ser facilmente calculadas com base na lei do inverso do quadrado da distância.

O fluxo solar é alterado consideravelmente durante o seu percurso entre o topo da atmosfera e a superfície terrestre pelas interações que ocorrem com os componentes da atmosfera através de processos de absorção e espalhamento da radiação incidente. 
Estima-se que apenas $25 \%$ da radiação incidente no TOA chegam à superfície terrestre sem sofrer nenhuma interação com os constituintes atmosféricos. Os $75 \%$ restantes são absorvidos, refletidos de volta ao espaço ou espalhados e, neste caso, normalmente atinge a superfície em uma direção diferente da direção de incidência no topo da atmosfera (LIOU, 2002).

A absorção atmosférica da irradiação solar ocorre em sua maior parte pela presença do ozônio e do vapor d'água, o dióxido de carbono, oxigênio e outros gases influenciam em menores proporções. O espalhamento ocorrido na atmosfera é causado sobretudo por particulados e gotículas de água.

A presença de nuvens reduz intensamente o fluxo incidente em até $85 \%$ devido à absorção e aos espalhamentos produzidos pelas gotículas de água. O espalhamento da irradiação solar pelas nuvens ocorre tanto na luz visível quanto no infravermelho próximo, sendo que a absorção é significativamente maior na faixa espectral do infravermelho próximo (Figura 2).

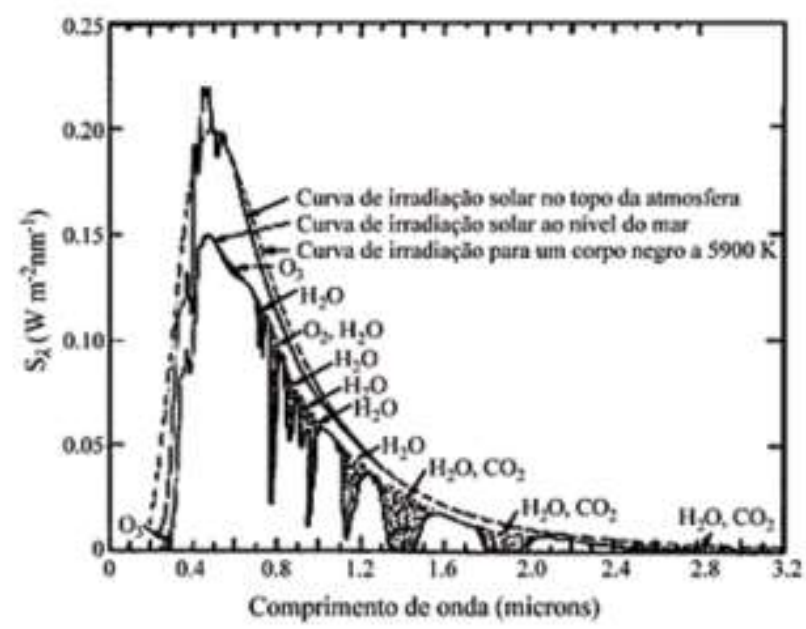

Figura 2. Espectro de radiação solar incidente no topo da atmosfera e na superfície da Terra e da emissão de um corpo negro a 5900 K. Fonte: MARTINS et al., 2004.

A intensidade da irradiação solar para uma localidade na superfície da Terra está relacionada não somente aos constituintes da atmosfera, como também à altitude relativa à proporção de massa de ar atravessada pela radiação e ao ângulo zenital solar ângulo formado entre a vertical e a linha do Sol, quanto maior esse ângulo, maior distância percorrida pela radiação e maior interferência da atmosfera (GUIMARÃES, 2003). 
Devido aos processos causados pela interferência da atmosfera, a irradiação solar incidente na superfície terrestre apresenta-se classificada em três formas:

- Irradiação Direta: definida como a fração da irradiação solar que atravessa a atmosfera terrestre sem sofrer alteração em sua direção original;

- Irradiação Difusa: refere-se à componente da irradiação solar que ao atravessar a camada atmosférica, é espalhada por elementos que constituem a atmosfera terrestre (moléculas de ar, aerossóis, poeira, etc.) e tem a sua direção de propagação alterada; - Irradiação Global: é a soma das componentes direta e difusa.

\subsection{Previsão da Radiação Solar}

O sistema de fornecimento de energia deve garantir o equilíbrio entre a produção e o consumo de energia elétrica a qualquer momento. Em muitos países, inclusive no Brasil, o uso de fontes de energia renováveis (solar, eólica, etc.) integrado ao sistema nacional de energia sofre com as características intrínsecas de variabilidade espacial e temporal da fonte energética, em alguns momentos este fato pode proporcionar um desequilíbrio na relação produção/consumo de eletricidade. A confiabilidade do sistema elétrico torna-se dependente da capacidade do sistema para acomodar as mudanças da relação produção/consumo de energia elétrica, ao mesmo tempo em que mantém a qualidade e a continuidade do serviço aos consumidores.

A intermitência e as características não-controláveis do recurso solar trazem algumas problemáticas, como: flutuações de tensão, qualidade da energia e questões de instabilidade. A previsão da radiação solar auxilia no gerenciamento e na programação do sistema, na indicação para armazenamento da energia excedente e também para a venda de ações no mercado de energia (VOYANT et al., 2017).

Um método eficiente de previsão ajudará a manejar as atividades dos operadores da rede elétrica, como identificar eventos repentinos, administrar as variabilidades das operações, realizar uma programação da transmissão de energia, apontar os momentos de restrição, possibilitar planejamentos e a otimização das instalações (DIAGNE et al., 2013).

Os modelos de previsão utilizados no setor de energia solar são classificados de acordo com os tipos de dados de entrada. Os modelos estatísticos utilizam dados de radiação solar observados em superfície e são aplicados para previsões de curtíssimo prazo ("nowcasting"), de 5 minutos até 6 horas (REIKARD, 2009). Para previsões a 
curto e longo prazo (de 6 horas em diante) são utilizados modelos numéricos de previsão do tempo alimentados por dados coletados em estações meteorológicas e dados de imagens de satélites (PEREZ et al., 2010).

Modelos estatísticos baseiam-se em dados históricos de irradiância solar e realizam uma análise estatística para obter a relação entre as varáveis de entrada e as variáveis de saída. São classificados em duas categorias: estatísticos e métodos de inteligência artificial. Os modelos auto regressivo (AR) de séries temporais, auto regressivo de média móvel (ARMA), modelos de regressões múltiplas são exemplos de métodos estatísticos. Os modelos de inteligência artificial incluem sistemas de lógica difusa, redes neurais artificiais (RNAs) e modelos de algoritmos genéticos. Para os modelos estatísticos, ainda são necessárias informações temporais sobre as condições de nuvens, que podem ser adquiridas através de imagens de satélites ou através de instrumentos que realizam fotografias do céu, estes são mais utilizados para previsões a curtíssimo prazo (menos de 1 hora), possuem uma melhor resolução espacial e temporal (DIAGNE et al., 2013).

Modelos numéricos de previsão do tempo são utilizados para realizar a previsão do tempo para até 15 dias adiante, baseiam-se na reprodução do fenômeno físico através da modelagem das condições atmosféricas utilizando as equações diferenciais que descrevem as leis físicas que regem a dinâmica atmosférica e os processos radiativos que nela ocorrem. Estes modelos predizem a probabilidade de formação de nuvens no local e também a radiação transmitida utilizando um modelo dinâmico da atmosfera (VOYANT et al., 2017). Para utilizar um modelo numérico de previsão do tempo é necessário obter informações das condições atuais da atmosfera. Esta informação é obtida através de uma rede de estações meteorológicas. As variáveis-chave são ventos, temperatura, umidade e pressão atmosférica (DIAGNE et al., 2013).

\subsection{Cadeias de Markov}

Um processo estocástico é uma coleção de variáveis randômicas indexadas por elementos t pertencente a um determinado intervalo de tempo. A cadeia de Markov é um processo estocástico caracterizado por seu estado futuro depender apenas do seu estado atual, sendo que os estados passados não influenciam no estado futuro. Se o espaço de estados é discreto (enumerável), então o modelo de Markov é denominado Cadeia de Markov (HOWARD, 1971; KONSTANTOPOULOS, 2009). 


\subsubsection{Definição da Cadeia de Markov}

Seja o processo estocástico $\left\{X_{n}, n=0,1,2, \ldots\right\}$ em tempo discreto. As variáveis $X_{n}$ possuem possíveis valores num conjunto $E$ finito ou infinito enumerável. $E$ chamase conjunto de estados ou espaço de estados. $X_{n}=i$ representa que o processo está no estado $i$ no tempo $n$. $X_{n}$ é cadeia de Markov se (HOWARD, 1971):

$$
\mathbb{P}\left[X_{n+1}=j \mid X_{n}=\boldsymbol{i}, X_{n-1}=i_{n-1}, \ldots, X_{0}=\boldsymbol{i}_{\mathbf{0}}\right]=\mathbb{P}\left[X_{n+1}=j \mid X_{n}=\boldsymbol{i}\right]=p_{i j}
$$

Desse modo, a chance de o processo estocástico estar no instante $n+1$ no estado $\mathrm{j}$ depende somente do estado no instante $n$, mas independe de toda a história do processo (estados $X_{0}, X_{1}, \ldots, X_{n-1}$ ). Esta propriedade chama-se propriedade markoviana. As probabilidades podem ser representadas através de matriz de transição:

$$
\left(\begin{array}{ccc}
p_{0,0} & \cdots & p_{0, n} \\
\vdots & \ddots & \vdots \\
p_{i, 0} & \cdots & p_{i, n}
\end{array}\right)
$$

\subsubsection{Método da Transformada Inversa}

O processo de modelagem de séries temporais foi baseado no método da transformada inversa para variáveis aleatórias discretas. Para simular amostras aleatórias de uma variável aleatória $X$ com função de distribuição $F$, isto é, queremos gerar uma variável aleatória $X$ que possui função de distribuição de probabilidade dada por:

$$
F_{x}(x)=\mathbb{P}\left[X=x_{j}\right]=p_{j}, j=0,1, \ldots, \sum_{j} p_{j}=1 \text { para todo } x .
$$

A função de distribuição de probabilidade pode ser representada pela matriz de probabilidade de transição. É gerada uma variável aleatória $U$ uniformemente distribuída em $[0,1]$, isto é, $U \sim U[0,1]$ e então, o método da transforma inversa estabelece que a variável aleatória $X=F_{X}^{-1}(U)$ (ROSS, 2013). Então,

$$
X=\left\{\begin{array}{c}
x_{0}, \text { se } U<p_{0} \\
x_{1}, \text { se } p_{0} \leq U<p_{0}+p_{1} \\
\vdots \\
x_{j}, \text { se } \sum_{i=0}^{j-1} p_{i} \leq U<\sum_{i=0}^{j} p_{i} \\
\vdots
\end{array}\right\}
$$

Portanto, se para $0<a<b<1, \mathbb{P}\{a \leq U \leq b\}=b-a$ temos que:

$$
\mathbb{P}\left\{X=x_{j}\right\}=\mathbb{P}\left\{\sum_{i=1}^{j-1} p_{i} \leq U \leq \sum_{i=0}^{j} p_{i}\right\}=p_{j}
$$


A implementação do método pode ser dada a seguir pelo algoritmo (ROSS, 2013):

1. Gerar um número aleatório $U$ de uma distribuição uniforme $[0,1]$;

2. Se $U<p_{0}$ fazer $X=x_{0}$ e parar por aqui;

3. Caso contrário, se $U<p_{0}+p_{1}$ fazer $X=x_{1}$ e parar por aqui;

4. Caso contrário, se $U<p_{0}+p_{1}+p_{2}$ fazer $X=x_{2}$ e parar por aqui;

5. $\vdots$ 


\section{OBJETIVOS}

Simulações realizadas com dados horários de irradiância negligenciam padrões de comportamento como picos ou reduções bruscas de energia solar em intervalos curtos de tempo, por consequência podem resultar em falsas predições de produção de energia. Esta pesquisa teve por objetivo criar um modelo para geração de uma série temporal de irradiância solar com valores de minuto a minuto, a partir de dados horários com finalidade de possibilitar uma melhor simulação da disponibilidade da energia solar e, consequentemente, da geração de eletricidade em projetos de exploração e comercialização a partir da conversão solar desta fonte. 


\section{MATERIAIS E MÉTODOS}

\section{1. Área de Estudo}

A região de estudo compreende a cidade de São Martinho da Serra no estado do Rio Grande do Sul. Esta área foi escolhida devido à disponibilidade de dados meteorológicos e radiométricos de minuto a minuto com registro histórico completo de 2005 a 2015.

A cidade de São Martinho da Serra situa-se na região central do estado do Rio Grande do Sul. A vegetação da região pertence à Floresta Estacional Decidual sendo uma zona transicional com os Campos Sul-rio-grandenses. O clima na cidade, assim como em toda a região sul do país, sofre influência da posição e intensidade da Alta Subtropical do Atlântico e sua circulação associada. Outra forte influência é o sistema de baixa continental (Baixa do Chaco), mais intenso no verão, que condiciona a circulação em baixos níveis e importantes fluxos de umidade e advecção de temperatura para a região Sul.

A precipitação em São Martinho da Serra é bem distribuída ao longo do ano. O maior número de dias com chuva ocorre durante os meses de inverno, porém o volume de precipitação é menor nesses dias, resultando em total mensal semelhante para todos os meses do ano. O total mensal médio anual da região fica entre 140 e 150mm. Durante os meses de junho, julho e agosto, as precipitações são mais frequentes e em menor quantidade. Mais de $50 \%$ das chuvas ocorridas nesses meses têm altura menor que $5 \mathrm{~mm}$.

A umidade relativa do ar mantém-se em média na faixa entre $65 \%$ e $75 \%$. Durante os meses de inverno, a umidade atinge seus picos mais elevados e depois cai gradativamente até atingir os $65 \%$, aproximadamente. A temperatura do ar máxima é de $24^{\circ} \mathrm{C}$, em média, e a temperatura mínima é de $14^{\circ} \mathrm{C}$, em média.

\subsection{Dados observados de irradiância solar}

Os dados de irradiância solar global utilizados neste estudo foram cedidos pelo Instituto Nacional de Pesquisas Espaciais (INPE), responsável pela operação das estações da rede SONDA (Sistema de Organização Nacional de Dados Ambientais). A rede SONDA é um projeto nacional para implementação de infraestrutura física e de recursos humanos destinada a levantar e melhorar a base de dados dos recursos de 
energia solar e eólica no Brasil. Os dados são coletados em conformidade com os padrões estabelecidos para a Baseline Surface Solar Radiation (BSRN). Além da aquisição de dados radiométricos, a estação coleta, de minuto a minuto, as informações meteorológicas (temperatura, umidade, pressão atmosférica, precipitação, direção e velocidade dos ventos, radiação solar) representativas da área em que está localizada (INMET, 2011).

Para o presente trabalho, foram utilizados dados de irradiância solar coletados na estação automática localizada em São Martinho da Serra - RS (29²6'34'S; 5349'23”W; $489 \mathrm{~m}$ de altitude). São registrados os valores médios de minuto a minuto de radiação global horizontal, radiação direta normal, radiação difusa, radiação de onda longa, iluminância, temperatura (superfície), umidade relativa, pressão atmosférica, precipitação de chuva, velocidade do vento $(10 \mathrm{~m})$ e direção do vento $(10 \mathrm{~m})$. Os dados são disponibilizados gratuitamente, em tempo real através do site http:ILsonda.ccst.inpe.br.

\subsection{Modelagem Numérica}

O presente trabalho teve como base o algoritmo de HOFMANN et al. (2014). O algoritmo consistiu em duas partes. A primeira parte foi um processamento dos dados brutos de entrada de irradiância global classificando-os e produzindo matrizes de transição de probabilidade (MTP) para três diferentes condições de tempo: céu claro, parcialmente nublado e nublado.

A segunda parte adotou o método da transformada inversa para variáveis aleatórias discretas para síntese de uma série temporal de irradiância global com resolução temporal de 1 minuto. Os dados de entrada consistiram nas matrizes de transição criadas na primeira etapa e nos dados de irradiância global. A ferramenta utilizada para o desenvolvimento dos códigos computacionais foi o Matlab ${ }^{\circledR}$.

\subsubsection{Matriz de Transição de Probabilidade (MPT)}

A classificação dos dados para as diferentes condições de tempo foi feita pelo índice de céu claro $\left(\boldsymbol{k}_{\boldsymbol{t}}\right)$. O índice $\boldsymbol{k}_{\boldsymbol{t}}$ é definido como a razão entre a irradiância global medida pela estação meteorológica e a irradiância global em condições de céu claro no mesmo local: 


$$
\boldsymbol{k}_{t}=\frac{E_{\text {medido }}}{E_{\text {céu claro }}}
$$

onde $\boldsymbol{k}_{\boldsymbol{t}}$ é o índice de céu claro, $E_{\text {medido }}$ é a irradiância medida no local e $E_{\text {céu claro }}$ é a irradiância para condições de céu claro.

O modelo de céu claro preconizado por Dumortier (1998) foi utilizado para

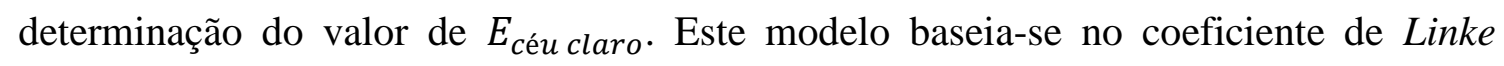
Turbidity (coeficiente de turbidez de Linke). Este parâmetro, em particular, é uma aproximação conveniente para modelar a absorção e espalhamento atmosférico da radiação solar sobre céu claro (LIMA, 2015). Descreve-se a espessura óptica da atmosfera devido tanto à absorção pelo vapor de água e à absorção e dispersão de partículas de aerossol em relação a um ambiente seco e limpo (KASTEN, 1996; DIABATÉ et al. 2003). Quanto maior for o valor do coeficiente de turbidez, maior a atenuação da radiação na atmosfera para céu claro.

A condição predominante de tempo num determinado dia resulta num padrão temporal característico de $\boldsymbol{k}_{\boldsymbol{t}}$ que pode ser utilizado para classificar aquele determinado dia numa das três classes (céu claro, parcialmente nublado, nublado). O algoritmo de detecção da condição de tempo é baseado no valor médio de $\boldsymbol{k}_{\boldsymbol{t}}$ do dia $(\bar{k} t$, dia $)$ e sua variabilidade durante o dia $(\tilde{k} t, d i a)$ :

$$
\begin{gathered}
\bar{k} t, d i a=\frac{1}{n} \sum_{i=1}^{24} k_{t, i} \\
\widetilde{k} t, d i a=\frac{1}{n} \sum_{i=1}^{24}\left|k_{t, i}-k_{t, i-1}\right|
\end{gathered}
$$

onde n é o número de horas em que a irradiância solar global de céu claro foi maior que $0 \mathrm{~W} / \mathrm{m}^{2}$, i.e., quando o Sol está acima do horizonte. A classificação foi feita de acordo com a Tabela 1.

Tabela 1. Visão geral das três classes de tempo e suas condições de detecção.

CLASSIFICAÇÃO DE NEBULOSIDADE

Nublado

Céu claro

Parcialmente nublado
CONDIÇÃO

$$
\begin{gathered}
0,6-\bar{k} t, \text { dia }>\tilde{k} t, \text { dia } \\
-0,72+0,8 \bar{k} t, \text { dia } \geq \tilde{k} t, \text { dia } \\
\text { Caso Contrário }
\end{gathered}
$$

Fonte: Adaptado de HOFMANN et al., 2014. 
Para cada classe de condição de nebulosidade foi criada uma matriz de probabilidade de transição. A MPT contém a informação de quão provável será a mudança de valor de $\boldsymbol{k}_{\boldsymbol{t}}$ num instante $i$ para outro valor no instante seguinte $i+1$. Para criar essas matrizes os dados medidos de irradiância a cada um minuto da mesma classe da condição de nebulosidade foram analisados e convertidos para uma matriz comum. As frequências para cada transição possível nos dados medidos foram registradas e posteriormente normalizadas para obter a MPT. Portanto, cada MPT contém todas as probabilidades de mudança de um valor específico $k_{t, i}$ para um valor $k_{t, i+1}$ do minuto $i$ para o seguinte de acordo com uma condição de nebulosidade específica.

\subsubsection{Série temporal}

A geração das séries de valores de $\boldsymbol{k}_{\boldsymbol{t}}$ com resolução de um minuto foi realizada pelo método da transformada inversa para variáveis aleatórias discretas. A MPT utilizada para realização do processo para um dado dia é escolhida em função da condição de nebulosidade do dia simulado. Estes são processos utilizados para modelar eventos reais, nos quais o primeiro estado não influencia o estado subsequente (eventos independentes).

Para determinar o sucessor $\boldsymbol{k}_{\boldsymbol{t}, \boldsymbol{i + 1}}$ de um valor específico de $\boldsymbol{k}_{\boldsymbol{t}}$ num determinado momento $i$, as probabilidades de que $\boldsymbol{k}_{t, \boldsymbol{i}+\boldsymbol{1}}$ seja igual a $\boldsymbol{k}_{\boldsymbol{t}, \boldsymbol{i}}$ foram acumuladas. Posteriormente um número aleatório entre 0 e 1 foi gerado e inserido como ponto limite na função de probabilidade cumulativa. O $\boldsymbol{k}_{\boldsymbol{t}, \boldsymbol{i}+\boldsymbol{1}}$ é definido no valor de $\boldsymbol{k}_{\boldsymbol{t}}$ em que a função de probabilidade é, pela primeira vez, maior que o número aleatório gerado. $\mathrm{O}$ processo foi continuado de maneira semelhante até gerar uma cadeia de 60 valores de $\boldsymbol{k}_{\boldsymbol{t}}$ por hora. A partir das sequências de $\boldsymbol{k}_{\boldsymbol{t}}$ os valores de irradiância para cada minuto foram calculados com o auxílio do modelo de céu claro ( $\left.\mathrm{E}_{\text {céu claro,i }}\right)$.

$$
E_{\text {gerado }, i}=\boldsymbol{k}_{t, i} \times E_{\text {céu claro }, i}
$$

Esse processo foi repetido, cada vez sorteando um número aleatório diferente, até que a média dos valores gerados a cada minuto fosse igual à média dos valores de irradiância usados como dado de entrada no período de uma hora ( $E_{\text {hora }}$ ) com a precisão desejada $(\delta=0,05)$. Os procedimentos mencionados foram calculados de acordo com a equação (7):

$$
\left|\frac{E_{\text {hora }}}{\sum_{i=1}^{60} E_{\text {gerado }, i}}-1\right|<\delta
$$




\subsection{Análises Estatísticas}

A geração das matrizes MPT foi realizada utilizando os dados de irradiância global observados na estação de São Martinho da Serra ao longo dos anos de 2013 e 2014. Os dados observados no ano de 2015 foram destinados como dado de entrada do modelo e para realizar a comparação e validação dos resultados obtidos pelo modelo estatístico.

Uma nova tabela do ano de 2015 foi construída apenas com dados horários instantâneos de irradiância ( 24 dados diários) para uso como dado de entrada, e a partir destes valores foi gerada a série temporal de 1 minuto de resolução. Os valores produzidos pelo modelo foram validados por meio de comparação estatística com os valores instantâneos de irradiância solar global observados (1440 dados diários) na estação.

\subsubsection{Variância}

Para o processo de validação, foram calculados os valores de variância, conforme descrito na equação (8), para os dados de irradiância global modelados pelo algoritmo e a variabilidade dos dados medidos utilizados para validação.

$$
\overline{\operatorname{Evar}}=\frac{1}{n} \sum_{i=2}^{n}\left|E_{i}-E_{i-1}\right|
$$

onde n representa o número de minutos em um dia (1440) e $\boldsymbol{E}_{\boldsymbol{i}}$ e $\boldsymbol{E}_{\boldsymbol{i}-\mathbf{1}}$ é o valor da irradiância global do dados modelados e/ou dados medidos para os instantes i e i-1.

\subsubsection{Frequência de gradientes}

Uma análise mais completa sobre a variabilidade pode ser obtida através do cálculo da frequência dos gradientes dos dados no período de 1 ano. $\mathrm{O}$ gradiente é a diferença absoluta entre o valor de irradiância global num determinado minuto para o seguinte e posteriormente é contabilizada a frequência dessas diferenças. O cálculo dos gradientes $(g)$ pode ser visualizado na equação (9):

$$
g=E_{i+1}-E_{i}
$$

Para realizar uma comparação entre os indicadores é realizado o cálculo da raiz do erro quadrático médio (RMSE em inglês) das frequências dos gradientes de irradiância global entre o dado modelado e o dado medido: 


$$
R M S E_{g}=\sqrt{\frac{1}{n_{g}} \sum_{1}^{n_{g}}\left[f i\left(g_{\text {modelo }, i}\right)-f i\left(g_{\text {medido }, i}\right)\right]^{2}}
$$

onde $R M S E_{g}$ é o erro quadrático médio das frequências dos gradientes, $n_{g}$ é o número total de gradientes observados e $f i\left(g_{\text {modelo, } i}\right)$ e $f i\left(g_{\text {medido, } i}\right)$ são as frequências dos gradientes de irradiância dos dados modelados e dos dados medidos, respectivamente.

\subsubsection{Frequência relativa de distribuição}

A frequência de distribuição de valores de irradiância é um indicador mais útil para a simulação de plantas de sistemas fotovoltaicos. Este teste calcula a frequência em que um valor específico de irradiância ocorre em um ano. A avaliação quantitativa dessas frequências também é feita através do cálculo do desvio médio quadrático. Cada valor $i$ de distribuição da irradiância no modelo é comparado com a distribuição no modelo, estes desvios são elevados ao quadrado e posteriormente somados e dividido pelo número de ocorrências. A raiz quadrada retorna o valor do $R M S E_{f d}$ :

$$
R M S E_{f d}=\sqrt{\frac{1}{n_{i}} \sum_{i=1}^{n_{i}}\left[f i\left(E_{\text {modelo }, i}\right)-f i\left(E_{\text {medido }, i}\right)\right]^{2}}
$$

onde $n_{i}$ é o número total de valores de irradiância observados, $f i\left(E_{\text {modelo,i }}\right)$ e $f i\left(E_{\text {medido, } i}\right)$ são as frequências dos valores de irradiância gerados pelo modelo e os valores medidos na estação meteorológica respectivamente.

\subsubsection{Teste de Kolmogorov-Smirnov}

O teste de Kolmogorov-Smirnov, ou simplesmente teste KS pode ser utilizado para verificar se a hipótese de que a distribuição dos dados modelados segue a mesma distribuição dos dados medidos. Este teste observa a máxima diferença absoluta entre a função acumulada assumida (dados medidos) e a função de distribuição empírica (dados modelados). Como critério, compara-se esta diferença com um valor crítico para um dado nível de significância (CRUTCHER, 1945; PES et al., 2017).

Calcula-se a máxima distância vertical entre os gráficos $F(x)$ e $F n(x)$ sobre a amplitude dos possíveis valores de $\mathrm{x}$. $\mathrm{O}$ valor $\mathrm{F}(\mathrm{x})$ representa a função de distribuição acumulada assumida para os dados e o valor $\mathrm{Fn}(\mathrm{x})$ representa a função de distribuição acumulada empírica dos dados:

$$
D_{n}=\left|F(x)-F_{n}(x)\right|
$$


A estatística do teste observa se o máximo valor de Dn é maior que o valor crítico, caso verdadeiro rejeita-se a hipótese de que a função de dados modelados segue a mesma distribuição que a função de dados observados com (1- $\alpha$ )100\% de confiança.

Para o presente trabalho utilizamos um nível de significância de 0,01, o cálculo do valor crítico (Vc) foi dado de acordo com a equação (14), onde n é o número total de valores de irradiância (CRUTCHER, 1945):

$$
V C=\frac{1,63}{\sqrt{n}}
$$




\section{RESULTADOS E DISCUSSÃO}

Os resultados foram baseados em três anos de dados da estação de São Martinho da Serra - RS, estes já estão qualificados e são disponibilizados gratuitamente pela rede SONDA. Dois anos foram usados para a criação das MPTs e para evitar auto referência, o ano subsequente foi destinado para validar o algoritmo desenvolvido.

A Tabela 2 contém um fragmento da MPT para a condição de céu nublado com os valores de probabilidade de mudança de estado do kt para a cidade de São Martinho da Serra. A título de exemplo, considerando a Tabela 2, a probabilidade do kt mudar de 0,1 para 0,09 num período de 1 minuto é de $19,97 \%$, a probabilidade de continuar 0,1 é de $48,31 \%$ e a probabilidade de mudar para 0,01 é de $0 \%$.

Tabela 2. Fragmento da MPT para a condição de céu nublado. Para cada valor de Kt no instante de tempo i (linhas), a probabilidade de mudança para outro valor de Kt no instante de tempo i+1 (colunas) é dado. Os valores de Kt variam de 0 a 1,5.

\begin{tabular}{|c|c|c|c|c|c|c|c|c|c|c|c|}
\hline \multirow{2}{*}{$\mathrm{Kt}, \mathrm{i}$} & \multicolumn{11}{|c|}{$\mathrm{Kt}, \mathrm{i}+\mathbf{1}$} \\
\hline & 0 & 0,01 & 0,02 & 0,03 & 0,04 & 0,05 & 0,06 & 0,07 & 0,08 & 0,09 & 0,1 \\
\hline 0 & 0,88560 & 0,11306 & 0,00135 & 0 & 0 & 0 & 0 & 0 & 0 & 0 & 0 \\
\hline 0,01 & 0,03030 & 0,89744 & 0,07071 & 0,00155 & 0 & 0 & 0 & 0 & 0 & 0 & 0 \\
\hline 0,02 & 0 & 0,06724 & 0,83526 & 0,09264 & 0,00336 & 0,00075 & 0,00037 & 0,00037 & 0 & 0 & 0 \\
\hline 0,03 & 0 & 0 & 0,10511 & 0,76170 & 0,12170 & 0,00809 & 0,00298 & 0,00043 & 0 & 0 & 0 \\
\hline 0,04 & 0 & 0 & 0,00356 & 0,13034 & 0,70463 & 0,14591 & 0,01201 & 0,00222 & 0,00089 & 0,00044 & 0 \\
\hline 0,05 & 0 & 0 & 0 & 0,00498 & 0,14764 & 0,66848 & 0,15580 & 0,01585 & 0,00543 & 0,00136 & 0 \\
\hline 0,06 & 0 & 0 & 0 & 0,00142 & 0,01614 & 0,16089 & 0,60465 & 0,17940 & 0,02943 & 0,00617 & 0,00142 \\
\hline 0,07 & 0 & 0 & 0 & 0,00045 & 0,00224 & 0,01520 & 0,16853 & 0,59186 & 0,18283 & 0,02772 & 0,00671 \\
\hline 0,08 & 0 & 0 & 0 & 0 & 0,00045 & 0,00134 & 0,02595 & 0,18479 & 0,55078 & 0,18389 & 0,04027 \\
\hline 0,09 & 0 & 0 & 0 & 0 & 0 & 0,00094 & 0,00612 & 0,02871 & 0,19671 & 0,50729 & 0,19671 \\
\hline 0,1 & 0 & 0 & 0 & 0 & 0 & 0 & 0,00185 & 0,00693 & 0,03375 & 0,19972 & 0,48313 \\
\hline
\end{tabular}

A previsão de irradiância para condições de céu nublado ou parcialmente nublado são mais difíceis quando comparado às condições de céu claro, devido à grande variabilidade das condições atmosféricas nos dois primeiros casos. A Figura 3 mostra exemplos de dias com as três condições de céu modeladas. Embora a ocorrência exata dos picos de irradiância do modelo estatístico não sejam coincidentes temporalmente, a variância dos valores modelados está de acordo com os dados medidos na estação meteorológica. Como exemplo, foi calculada a variância do dia 03 de setembro de 2015 (Figura 3 (b)). O modelo mostrou uma variância de $60 \mathrm{~W} / \mathrm{m}^{2}$, enquanto o dado medido teve uma variância de $54 \mathrm{~W} / \mathrm{m}^{2}$. 

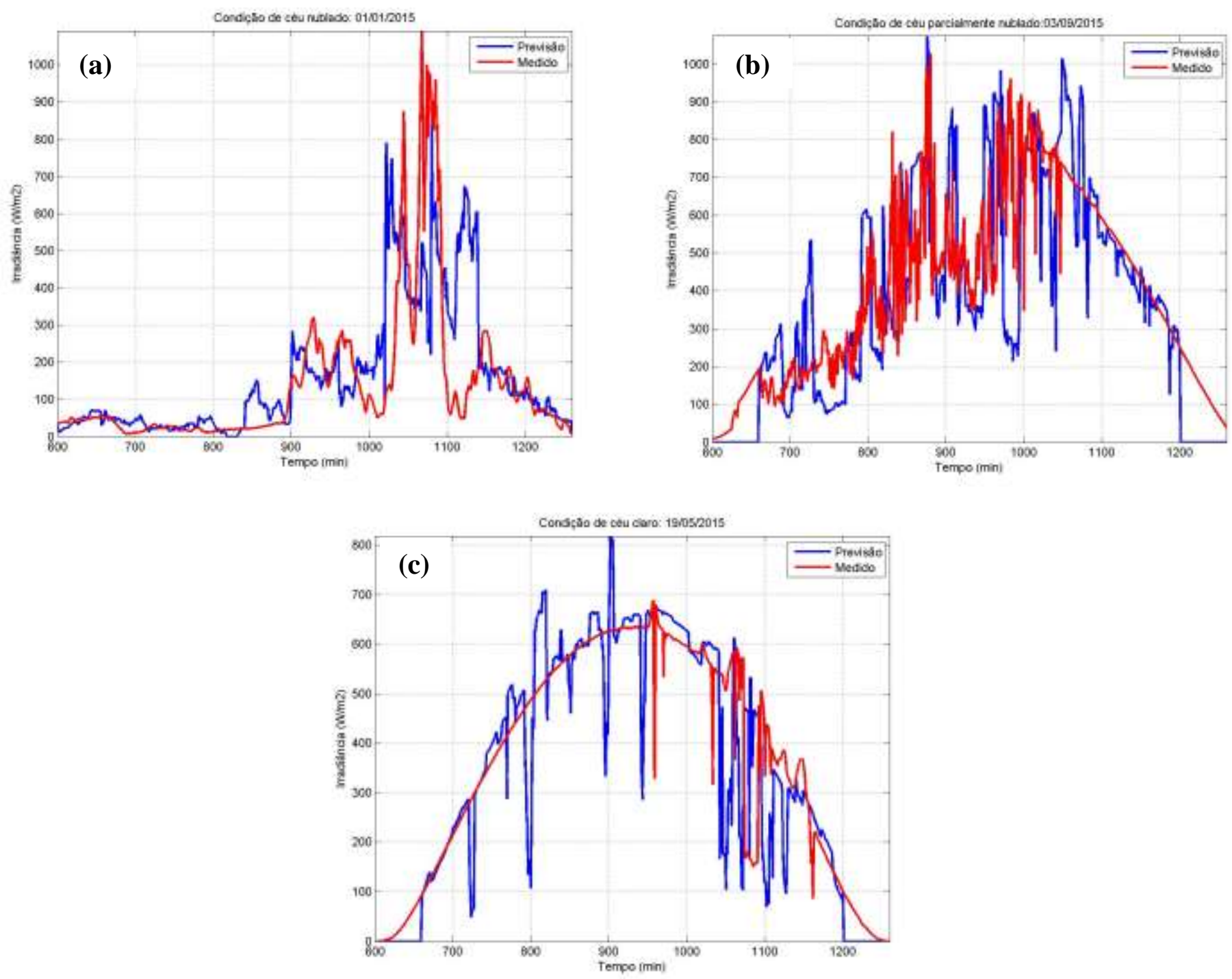

Figura 3. Curso temporal da irradiância global para três condições de tempo diferentes. (a) Condição de céu nublado. (b) Condição de céu parcialmente nublado. (c) Condição de céu claro. A linha vermelha corresponde a valores de irradiância global medidos (dado de validação) e a linha azul corresponde a valores de irradiância global gerados a partir do modelo estatístico desenvolvido neste trabalho.

Ao analisar os gráficos na Figura 3(b) e (c) é possível observar que para períodos muito próximos ao nascer e pôr-do-sol o modelo não gerou dados de irradiância. Nestes períodos os valores de $k_{t}$ calculados foram maiores que 1,5 , estes valores foram desconsiderados, pois o modelo de céu claro não faz uma boa representação do valor físico nestes instantes.

Para analisar a variabilidade da irradiância solar de uma forma mais completa pode-se calcular a frequência dos gradientes para o ano todo. Os gradientes são as diferenças absolutas dos valores de irradiância de um minuto para o próximo para os dados medidos e para os dados modelados. A Figura 4 mostra a frequência dos 
gradientes de irradiância para São Martinho da Serra no ano de 2015. Para gradientes entre 10 e $100 \mathrm{~W} / \mathrm{m}^{2}$ o modelo foi capaz de reproduzir uma distribuição similar a dos dados medidos. Para desvios acima de $100 \mathrm{~W} / \mathrm{m}^{2}$ o modelo, de uma maneira geral subestimou os dados medidos.

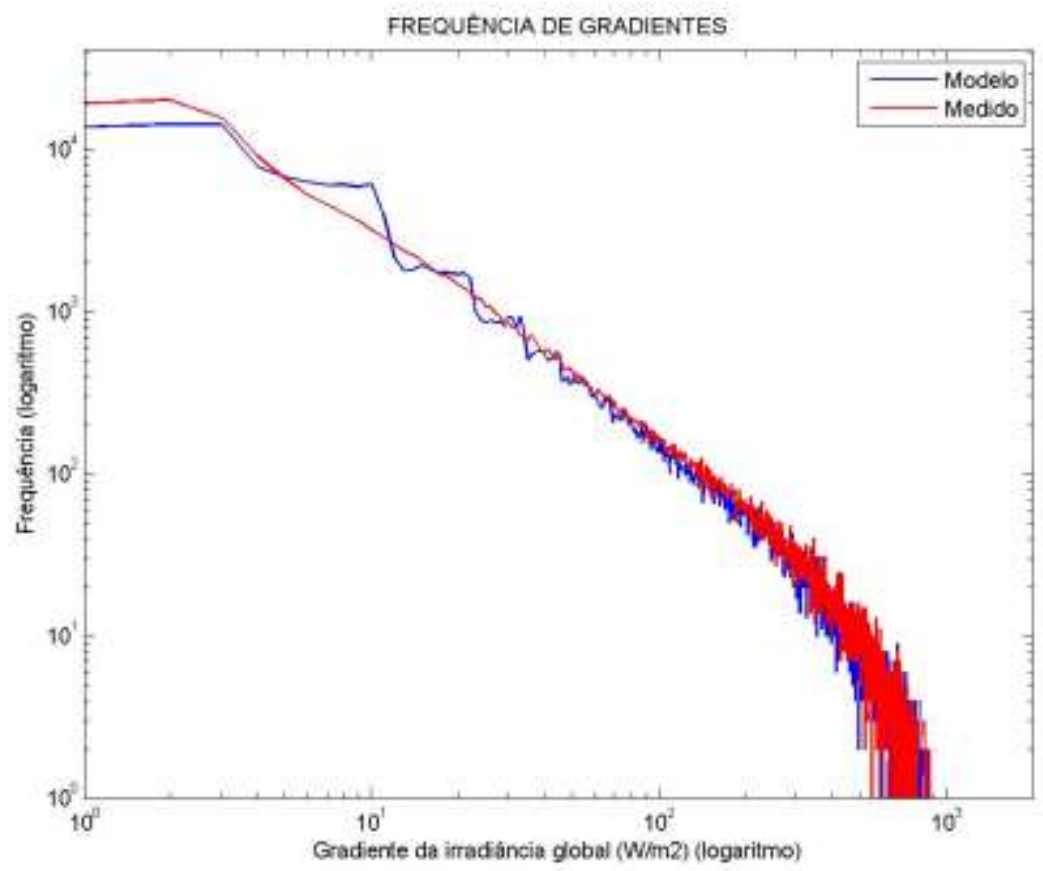

Figura 4. Frequência de gradientes da irradiância global em São Martinho da Serra - RS. A linha azul corresponde a frequência de gradientes gerados pelo modelo estatístico e a linha vermelho corresponde a frequência dos dados medidos na estação.

O RMSE representa uma medida de dispersão entre valores observados e modelados e é usado para indicar a acurácia dos resultados obtidos, quanto mais próximo de zero, mais preciso. Quando calculado o $R M S E_{g}$ da frequência de gradientes, o modelo aqui desenvolvido apresentou valores inferiores inclusive ao trabalho base desenvolvido por Hofmann et al. (2014) para a cidade de Lindenberg na Alemanha com dados de 2005 e aos modelos comparados neste mesmo artigo, desenvolvidos por Aguiar \& Collares-Pereira (1992) e Skartveit \& Olseth. (1992) para a mesma cidade, conforme demonstrado na Tabela 3. 
Tabela 3. Desvios médios quadráticos (RMSE) das frequências de distribuição de gradientes de irradiância e das frequências relativas de distribuição em porcentagem dos dados modelados quando comparados aos dados medidos.

\begin{tabular}{lcc}
\hline \multicolumn{1}{c}{ Modelo } & $\begin{array}{c}\text { RMSE da frequência de } \\
\text { gradientes }\end{array}$ & $\begin{array}{c}\text { RMSE em \% da } \\
\text { frequência relativa de } \\
\text { distribuição }\end{array}$ \\
\hline Aguiar \& Collares-Pereira & 8131 & 0,53 \\
Skarveit \& Olseith & 4758 & 0,68 \\
Hofmann et al. & 2787 & 0,21 \\
Soares \& Martins & 470 & 0,015 \\
\hline
\end{tabular}

A frequência relativa de distribuição anual dos valores de irradiância global pode ser observada na Figura 5. Este gráfico mostra o quão frequente um determinado valor de irradiância ocorre durante o período de 1 ano. Os maiores valores de irradiância geralmente ocorrem em dias de céu claro. Valores mais baixos $\left(0\right.$ a $\left.200 \mathrm{~W} / \mathrm{m}^{2}\right)$ e mais altos $\left(>1000 \mathrm{~W} / \mathrm{m}^{2}\right.$ ) foram superestimados, enquanto valores intermediários (200 a 1000 $\mathrm{W} / \mathrm{m}^{2}$ ) foram subestimados.

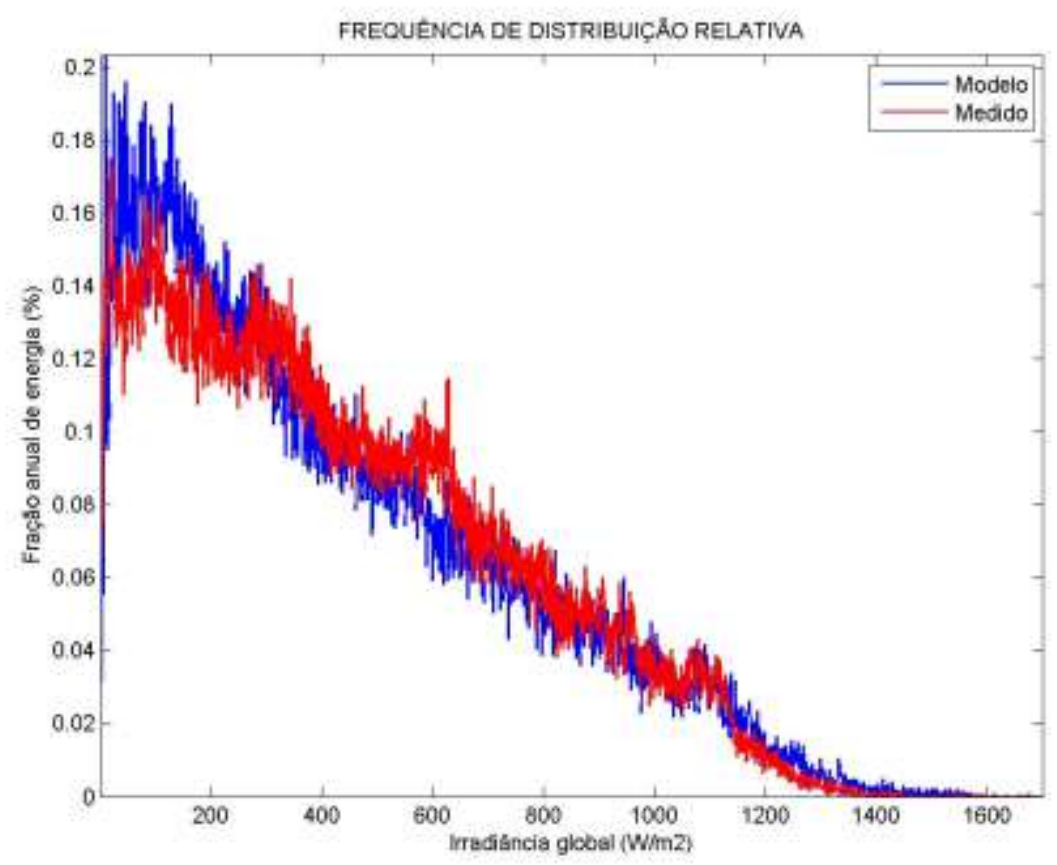

Figura 5. Frequência anual de distribuição relativa da irradiância global. Linha em azul representa os dados modelados e a linha em vermelho representa o dado medido.

O indicador mais correto para analisar quantitativamente o gráfico da Figura 5 é o cálculo do $R M S E_{f d}$ dessa distribuição, este resultado pode ser encontrado na Tabela 3. O resultado encontrado foi bastante superior quando comparado aos trabalhos de Hofmann et al. (2014), Aguiar \& Collares-Pereira (1992) e Skartveit \& Olseth. (1992). 
A possível razão para este fato pode ser explicada pela forma como foi produzida a MPT. Diferente dos outros trabalhos, a criação da MPT no presente trabalho envolveu dados de apenas uma localidade: São Martinho da Serra - RS com 2 anos de coleta de dados. No trabalho de Hofmann et al. (2014) as MPTs criadas envolviam múltiplas estações meteorológicas de diversas localidades, inclusive diversos países. Apesar de ser um número maior de dados, estes não pareceram gerar resultados tão precisos para qualquer região.

Outro fator que pode ter influenciado positivamente nos resultados foi a escolha do modelo de céu claro tanto para o cálculo do kt (Equação 3), como para gerar os valores de irradiância modelados (Equação 6). No presente trabalho foi utilizado o modelo de céu claro de Dumortier (1998) que utiliza o coeficiente de turbidez de Linke. A escolha deste modelo se deu a partir de uma análise inicial dos valores de kt no processo de elaboração do algoritmo. $\mathrm{O}$ índice $k_{t}$ idealmente não deve ser maior que 1 , já que a condição de céu claro indica a condição na qual há a menor atenuação da radiação solar pela atmosfera. O modelo de céu claro utilizado por Hoffman et al. (2014) em seu trabalho foi o de Bourges (1979), este modelo foi testado e não gerou bons resultados.

O teste Kolmogorov-Smirnov foi utilizado para verificar a hipótese de que a frequência de distribuição acumulada dos dados modelados segue a mesma distribuição que os dados medidos. O cálculo da máxima diferença mostrou-se menor que o valor crítico, portanto a hipótese não foi rejeitada. As frequências de distribuição acumuladas podem ser visualizadas na Figura 6. 


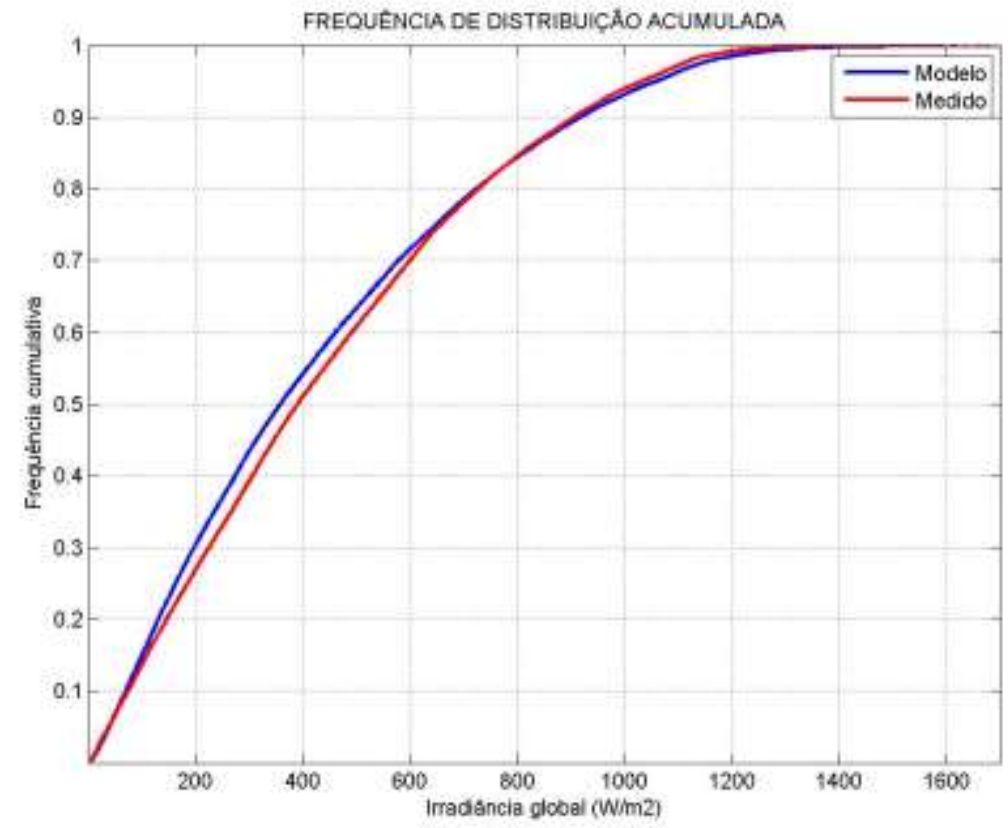

Figura 6. Frequência de distribuição acumulada anual. Em azul encontram-se os dados do modelo estatístico desenvolvido e em vermelho encontram-se os dados medidos na estação meteorológica. 


\section{CONSIDERAÇÕES FINAIS}

O modelo estatístico de previsão de irradiância global de minuto a minuto mostrouse eficiente quando comparado aos dados utilizados para validação. Apresentou resultados superiores ao modelo desenvolvido por Hofmann et al. (2014), trabalho este utilizado como base para o desenvolvimento do algoritmo. Quando realizado o teste de hipótese de Kolmogorov-Smirnov, o resultado obtido mostrou-se positivo, sendo a hipótese de que as duas curvas (dados modelados e dados medidos) apresentam a mesma distribuição cumulativa de probabilidade não rejeitada.

O modelo apresenta a vantagem de ser de simples execução e sem necessidade de computadores com alta capacidade de processamento. Pode ser desenvolvido em qualquer ferramenta computacional, neste caso foi utilizado o Matlab, mas pode ser desenvolvido em uma plataforma gratuita como o Python.

O modelo estatístico aqui desenvolvido combina as vantagens da maior simplicidade no desenvolvimento do código, além de realizar uma diferenciação das condições de tempo, fornecendo valores mais precisos e capazes de reproduzir com confiabilidade a variabilidade temporal real de cada localidade.

A escolha do modelo de céu claro de Dumortier (1998) pareceu influenciar positivamente nos resultados, evidenciando que uma análise criteriosa dos valores de $k_{t}$ calculados deve ser realizada. Além disso, a criação de MPTs a partir de uma única localidade para gerar os dados do modelo também pareceu influenciar positivamente nos resultados.

Os resultados deste trabalho podem atender a diversos setores da economia brasileira, como as concessionárias, geradoras e distribuidoras de energia elétrica e ao Operador Nacional do Sistema Elétrico ao reproduzir com alta confiança estatística os padrões de variabilidade da irradiância solar, possibilitando a simulação e o planejamento de plantas fotovoltaicas mais precisas.

Como sugestão de trabalhos futuros, o modelo estatístico desenvolvido no presente trabalho deve ser testado em outras localidades, a fim de verificar seu desempenho com outros dados e conferir a reprodutibilidade do algoritmo. 


\section{REFERÊNCIAS BIBLIOGRÁFICAS}

AGUIAR, R.; COLLARES-PEREIRA, M. TAG: a time-dependent, autoregressive, Gaussian model for generating synthetic hourly radiation, Solar Energy, v. 49, n. 6, p. 167-174, 1992.

BOURGES, G. Reconstitution des courbes de frequénce cumulées de l'irradiation solaire globale horaire recue par une surface plane, Report CEE 295-77, ESF of Centre d'Energétique de l'Ecole Nationale Supérieure des Mines de Paris, tome II, Paris, France, 1979.

DIABATÉ, L. REMUND , J. WALD, L. Linke turbidity factors for several sites in Africa Solar Energy, v.75, p.111-119, 2003.

CRUTCHER, H.L. A note on possible misuse of the Kolmogorov-Smirnov test. Journal of Applied Meteorology, v. 14, p. 1600-03, 1975.

DIAGNE, M; DAVID, M.; LAURET, P.; BOLAND, J.; SCHMUTZ, N. Review of solar irradiance forecasting methods and a proposition for small-scale insular grids. Renewable and Sustainable Energy Reviews, v.27, p. 65-76, 2013.

DUMORTIER D. The Satellight model of turbidity variations in Europe. In: SATELLIGHT MEETING, 6., 1998, Freiburg, Germany. Proceedings. Freiburg, 1998. Technical Report.

EPE. Análise da Inserção da Geração Solar na Matriz Elétrica Brasileira. Rio de Janeiro, 2012.

GUIMARÃES, A.P.C. Estimativa de Parâmetros da Camada Atmosférica para Cálculo da Irradiação Solar Incidente na Superfície Terrestre - Tese de Doutorado, COPPE/UFRJ, Rio de Janeiro, 2003.

HEINEMANN, D; LORENZ, E; GIRODO, M. Forecasting of solar radiation. Solar energy resource management for electricity generation from local level to global scale.

Nova Science Publishers, New York, 2006.

HOFMANN, M.; RIECHELMANN, S.; CRISOSTO, C.; MUBARAK, R.; SECKMEYER, G. Improved Synthesis of Global Irradiance with One-Minute Resolution for PV System Simulations. International Journal of Photoenergy, 2014. HOWARD, R.A. Dynamic Probabilistic Systems, JohnWiley \& Sons, v.1, New York, NY, USA, 1971. 
INMET. Informações sobre amostragem e parâmetros medidos por uma Estação Meteorológica Automática $\quad$ (EMA). NOTA TÉCNICA Nº01/2011/SEGER/LAIME/CSC/ INMET, 2011. Disponível em: < http://www.inmet.gov.br/portal/css/content/topo_iframe/pdf/Nota_TecnicaRede_estacoes_INMET.pdf> Acesso em: 13 Out 2017.

IQBAL, M. An Introduction to Solar Radiation. Toronto: Academic Press Canada, 1983.

KASTEN, F. The Linke turbidity factor based on improved values of the integral Rayleigh optical thickness. Solar Energy, v.56, n.3, p.239-44, 1996.

KONSTANTOPOULOS, T. Introductory lecture notes on MARKOV CHAINS AND RANDOM WALKS, 2009. Disponível em:

http://www2.math.uu.se/ takis\%20/L/McRw/mcrw.pdf Acesso em: 21 Jun 2018.

LIMA, F.J.L. Previsão de irradiação solar no nordeste do Brasil empregando o modelo WRF ajustado por Redes neurais artificiais (RNAS). Tese de Doutorado INPE, São José dos Campos, 2015.

LIOU, K. N. Radiation and cloud processes in the atmosphere: Theory, observation and modeling. New York: Oxford, 1992. p.486.

LORENZ, E.; HAMMER, A.; HEINEMANN, D. Short term forecasting of solar radiation based on satellite data. In: EUROSUN2004 (ISES Europe Solar Congress), p.841-8, 2004.

MARTINS, F.R.; PEREIRA, E.B.; ECHER, M.P.S. Levantamento dos recursos de energia solar no Brasil com o emprego de satélite geoestacionário - o Projeto Swera. Revista Brasileira de Ensino de Física, v. 26, n. 2, p. 145 - 159, 2004.

PES, M.P; PEREIRA, E.B.; MARENGO, J.A.; MARTINS, F.R.; HEINEMANN, D.; SCHMIDT, M. Climate trends on the extreme winds in Brazil. Renewable Energy, v. 109, p.110-120, 2017.

ROSS, S. M. Generating Discrete Random Variables. In: ROSS, S.M. Simulation. San Diego: Academic Press, 2013. p. 47-68.

SKARTVEIT, A.; OLSETH, J.A. The probability density and autocorrelation of shortterm global and beam irradiance, Solar Energy, v.49, n.6, p. 477-487, 1992.

PEREZ, R.; KIVALOV, S.; SCHLEMMER, J.; HEMKER JR, K.; RENNE, D.; HOFF, T.E. Validation of short and medium term operational solar radiation forecasts in the US. Solar Energy, v.84, p.2161-72, 2010. 
REIKARD, G. Predicting solar radiation at high resolutions: a comparison of time series forecasts. Solar Energy, v.83, p.342-9, 2009.

VOYANT, C.; NOTTON, G.; KALOGIROU, S.; NIVET, M.L.; PAOLI, C.; MOTTE, F.; FOUILLOY, A. Machine learning methods for solar radiation forecasting: A review. Renewable Energy, v.105, p.569-582, 2017. 